\title{
Physical origin of directional beaming emitted from a subwavelength slit
}

\author{
Liang-Bin Yu, ${ }^{1}$ Ding-Zheng Lin, ${ }^{1}$ Yi-Chun Chen, ${ }^{1}$ You-Chia Chang, ${ }^{1}$ Kuo-Tung Huang, ${ }_{1}^{1}$ Jiunn-Woei Liaw, ${ }^{2}$ \\ Jyi-Tyan Yeh, ${ }^{2}$ Jonq-Min Liu, ${ }^{2}$ Chau-Shioung Yeh, ${ }^{1}$ and Chih-Kung Lee ${ }^{1,3,4, *}$ \\ ${ }^{1}$ Institute of Applied Mechanics, National Taiwan University, Taipei, Taiwan \\ ${ }^{2}$ Materials Research Laboratory, Industrial Technology Research Institute, Hsinchu, Taiwan \\ ${ }^{3}$ Institute of Engineering Science and Ocean Engineering, National Taiwan University, Taipei, Taiwan \\ ${ }^{4}$ Department of Engineering and Applied Sciences, National Science Council, Taiwan
}

(Received 16 September 2004; published 19 January 2005)

\begin{abstract}
We propose the physical origin for a directional beam of light emitting from a single subwavelength slit in metallic film that is characterized by a corrugation feature at the exiting side of the film. We theorize that the beaming phenomenon can be explained simply as surface plasmon diffraction along the corrugation as long as the multiple scattering effects are taken into account to restate the dispersion relationship of the surface plasmon. In order to prove our theory, both an experimental setup and numerical simulations were undertaken. Results obtained match well with our theory of an explanation based on a surface plasmon diffraction scheme.
\end{abstract}

DOI: 10.1103/PhysRevB.71.041405

PACS number(s): 78.66.Bz, 42.25.Fx, 42.79.Dj, 73.20.Mf

The phenomenon dealing with extraordinary transmission has stimulated much interest over the years. ${ }^{1,2}$ In one study, it was found that when we illuminated a metallic film perforated with periodic subwavelength apertures, the transmission was unexpectedly large in certain wavelength regions. Another study, based on an astounding phenomenon called "directional beaming" recently published by Ebbesen et al., ${ }^{3}$ found that under transverse magnetic polarization illumination, when a single slit in a metallic screen is surrounded by periodic surface corrugations, the emitted light field from the slit will concentrate in a specific direction at a confined angle, instead of diverging in all directions. This phenomenon was confirmed experimentally in another study using microwaves. ${ }^{4}$ Some theoretical methods by means of Huygens's principle and surface cavity resonance recently published ${ }^{5-7}$ provide information to predict the beaming phenomenon. However, the underlying physical mechanism is still not clear and is still being investigated by many. Herein, we propose a comprehensive explanation to the intriguing beaming phenomenon. Our theory is based on the diffraction of surface plasmon (SP) propagating along the corrugation beside the slit. We validated this theory both experimentally and numerically with an excellent match of the two results.

The beaming structure discussed is composed of a subwavelength aperture in a metallic thick film with surface gratings on the exiting plane (Fig. 1). We used finitedifference time-domain (FDTD $)^{8}$ simulations and observed that the incident $p$-polarization electromagnetic waves induced the surface plasmon and caused flow along the metallic surface through the aperture where it is then diffracted by the periodic corrugation on the exiting side. Then, the socalled grating diffraction takes place and the beaming phenomenon can be observed. However, if we use a surface plasmon dispersion relationship [Eq. (1)] along an infinite planar interface between a dielectric and metal $^{9}$ and take the grating equation [Eq. (2)] to calculate the diffracted angle, we get the following:

$$
\begin{gathered}
k_{s p}=k_{0} \sqrt{\frac{\varepsilon_{\text {dielectric }} \cdot \varepsilon_{\text {metal }}}{\varepsilon_{\text {dielectric }}+\varepsilon_{\text {metal }}}}, \\
k_{s p} \pm m \frac{2 \pi}{\Lambda}=k_{0} \sin \theta,
\end{gathered}
$$

where $k_{s p}$ represents the wave vector of SP, $k_{0}$ the wave vector in free space, and $\Lambda$ the grating period; no desirable prediction can be found to match the experimental results in Ref. 3. This result indicates that a surface plasmon wave vector larger than that in Eq. (1) will be required to support the above diffraction argument. In fact, this is the case when the surface plasmon dispersion relationship is altered by surface corrugation/roughness, especially when the depth of surface corrugation is not negligible. ${ }^{10}$ This is known as a multiple scattering effect. Therefore, in this case, Eq. (1) is not applicable, and we need to identify the actual dispersion formula of the surface plasmon.

The existence of surface plasmon is typically recognized to be the reason behind a grating resonance anomaly, ${ }^{11-13}$ resulting in a bell-like variation in the spectrum signal that signifies a maximum absorption of an external incident energy. This resonance phenomenon provides us with a physical path to approach the needed dispersion formula. Although the way to calculate the dispersion relation is clear, in general, its analytical expression is difficult to derive. We can try to obtain it numerically instead. We adopt the concept of a Helmholtz reciprocity theorem ${ }^{14}$ and chose a rigorous coupled wave analysis (RCWA) method ${ }^{15}$ to deal with the computations. Therefore, the beaming angles can be obtained from the angles excited on the surface plasmon, which correspond to a minimum reflection or maximum absorption. [Fig. 1(a)].

In summary, we propose a SP diffraction scheme to explain the beaming phenomenon where we believe the beaming angle can be successfully predicted. In addition, we can convert the original directional beaming for obtaining the resonance condition of the surface corrugation/grating by lo- 


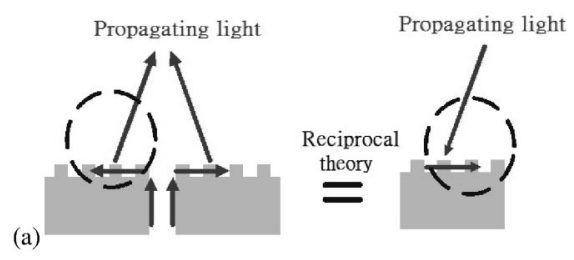

(b)
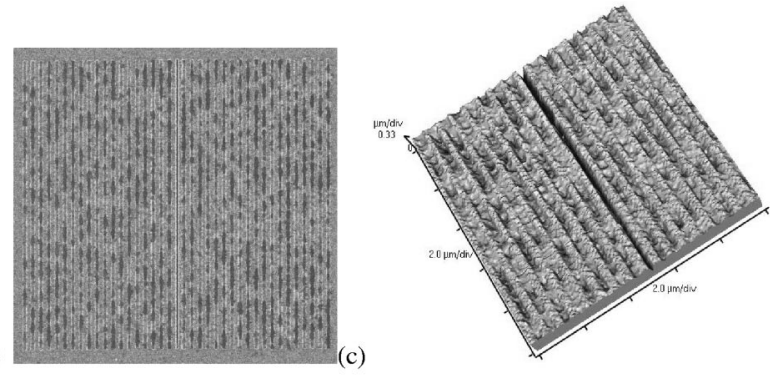

FIG. 1. (a) Concept of Helmholtz's reciprocity theorem. (b) SEM and (c) AFM image of the beaming structure. (The structure is composed of an opaque silver film perforated by a slit with a surface grating on the exiting side.)

cating the maximum energy absorption. In order to prove our theory, both experimental ${ }^{16}$ and numerical analyses were performed. We considered a sample with a surface binary grating fabricated on a 200-nm-thick Ag film, where the nominal

\section{Red $(633 \mathrm{~nm}) \quad$ Green $(520 \mathrm{~nm}) \quad$ Blue $(467 \mathrm{~nm})$}

(a)
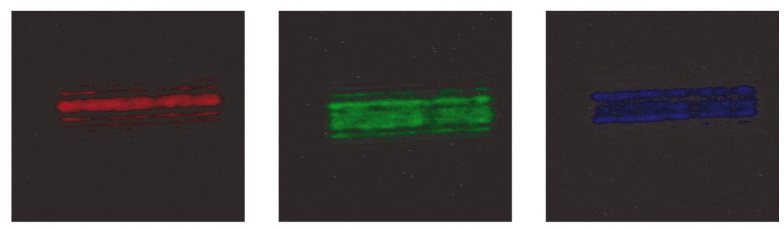

(b)
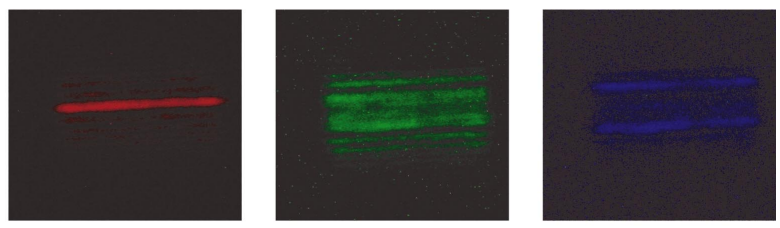

(c)
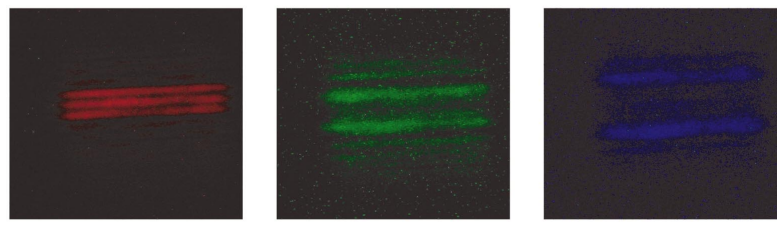

(d)
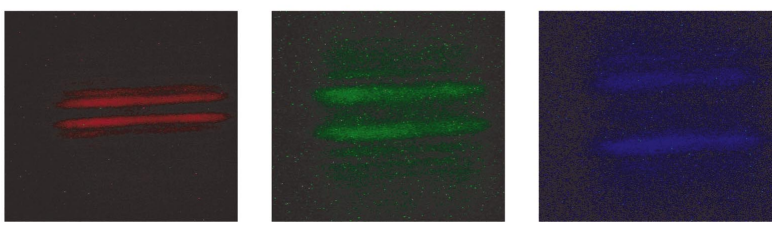

FIG. 2. (Color) Optical images at a distance above the surface structure: (a) 4, (b) 8, (c) 12, and (d) $16 \mu \mathrm{m}$.

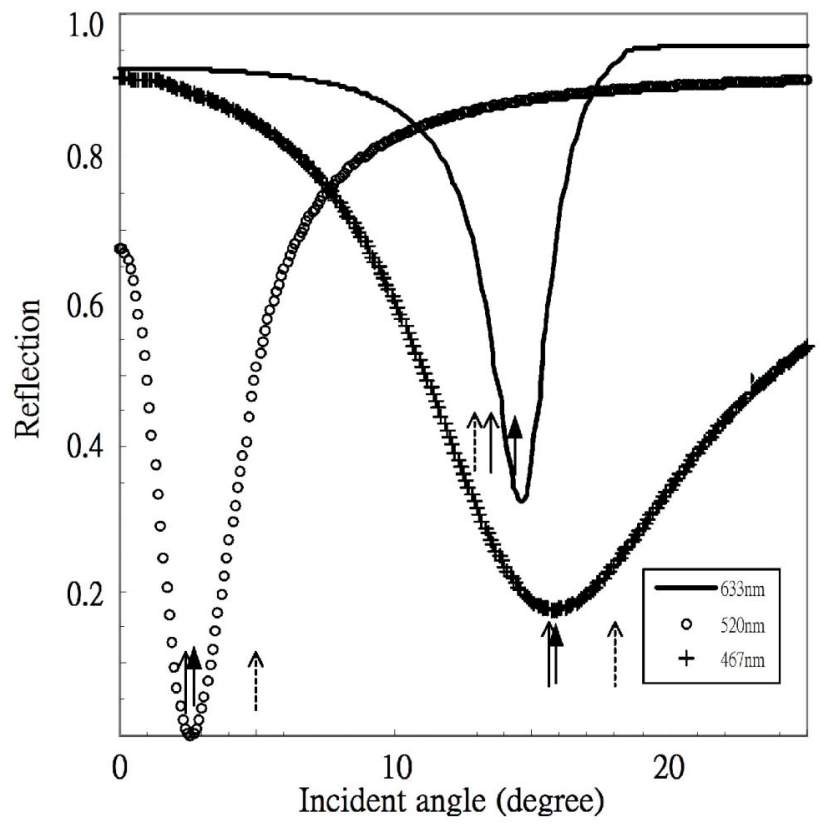

FIG. 3. Beaming angle calculation results based on RCWA simulations [the minimum reflection (maximum absorption) indicates the beaming angle]. The refraction indices are from calculations in Ref. 17. The arrows indicate the location of the beaming angle by FDTD simulation ( $\uparrow$ ), RCWA simulation $(\boldsymbol{\uparrow})$, and experimental results $(\uparrow)$.

grating period, grating depth, and duty cycle were set as $480 \mathrm{~nm}, 40 \mathrm{~nm}$, and 0.5 , respectively. The fabrication process then took place. The first step uses optical lithography to pattern a target crossmark that was then transferred to a quartz substrate with a reactive ion etching system. Then, a 200-nm silver film was deposited onto the substrate using a thermal evaporator. Finally, the surface structures needed were patterned on the silver film by using a focus ion beam (FIB) system. The final beaming structure, developed as seen by scanning electron microscopy (SEM) and atomic force microscopy (AFM) metrology [shown in Figs. 1(b) and 1(c)], was characterized by an actual grating period, grating depth, and duty cycle at $481 \mathrm{~nm}, 42 \mathrm{~nm}$, and 0.5 , respectively. An inverted microscope equipped with a transmission halogen light source with a $10-n m$ bandpass color filter (633, 520 , and $467 \mathrm{~nm}$ ) was used to determine the beaming angles. Photographs of the transmitted light beam images were taken at every single micrometer above the sample surface by using an attached charge-coupled device (CCD) camera.

The optical images shown in Figs. 2(a)-2(d) reflect the images taken at a distance off the exit surface of the optical structure from 4 to $16 \mu \mathrm{m}$. The various varying concentrated beaming situations for each wavelength can be seen. After specifying the lateral location of the emerging beams, we can derive the regression lines and estimate the beaming angles via the slope of the lines. The beaming angle appears at $12.7^{\circ}$ at $633 \mathrm{~nm}$ incident light, $2.3^{\circ}$ at $520 \mathrm{~nm}$, and $15.7^{\circ}$ at $467 \mathrm{~nm}$. On the other hand, according to our theory, we can obtain the surface plasmon dispersion relationship for each wavelength by calculating the minimum total diffraction efficiency outside the grating structure (Fig. 3). By a reverse of 


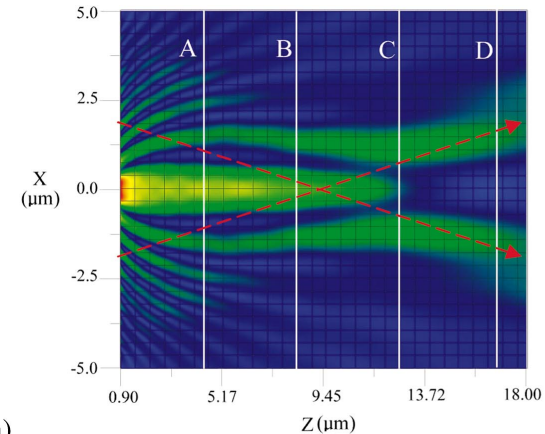

(a)

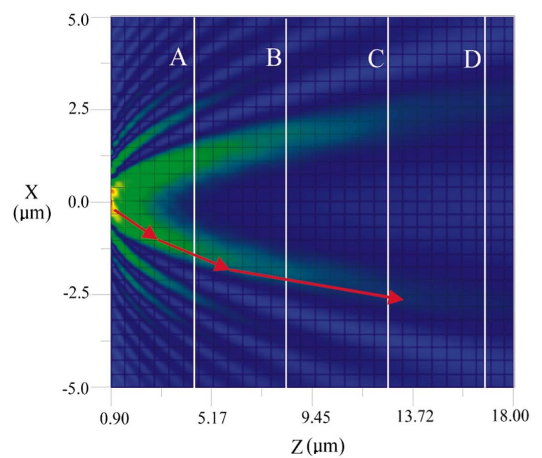

(d)

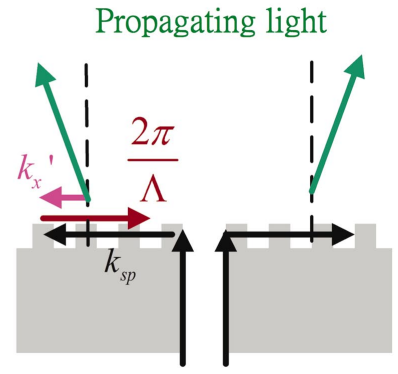

(b)

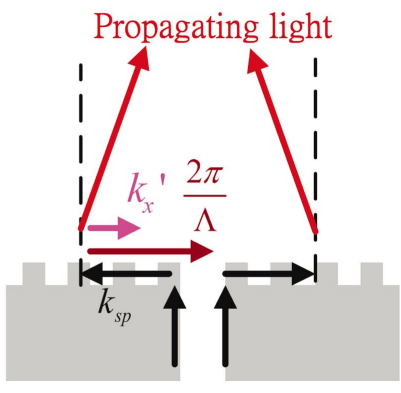

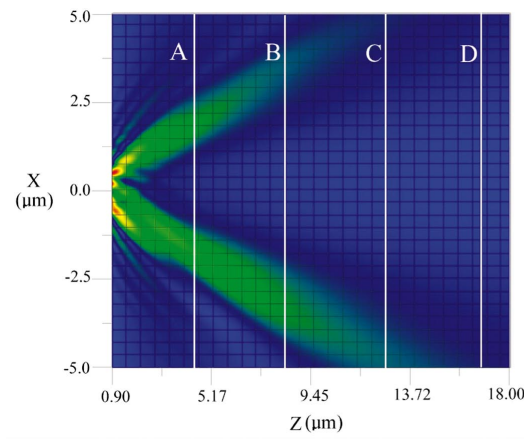

FIG. 4. (Color) Calculated energy distribution and angular identification of the transmission field. The illumination wavelength is $633 \mathrm{~nm}$ in (a) and (b), $520 \mathrm{~nm}$ in (c) and (d), and $467 \mathrm{~nm}$ in (e) and (f). Note (a), (c), and (e) are the transmitted energy distributions calculated by FDTD. The solid lines A-D represent the cross area at $4,8,12$, and $16 \mu \mathrm{m}$ above the surface structure; while (b), (d), and (f) show the sign convention of the diffracted angles. (e)

the wave, we can observe that the resonance angles are actually equal to the diffraction angles, i.e., the beaming angles. Our theoretical predications of $14.6^{\circ}, 2.6^{\circ}$, and $15.8^{\circ}$ at 633,520 , and $467 \mathrm{~nm}$ illumination, respectively, closely matches the experimental data. Thus we are convinced that a surface plasmon diffraction scheme is capable of explaining the directional beaming phenomenon.

To delve further into the unusual beaming nature, FDTD simulations were carried out and are illustrated in Fig. 4. It should be noted that a remarkable characteristic was observed [Fig. 4(a)]. Under a $633 \mathrm{~nm}$ illumination, the light appears in the center at a distance $4 \mu \mathrm{m}$ above the exit surface (slice A). The beam appears to propagate without angular divergence but on the contrary, its central beam width seems smaller than the prior case at $8 \mu \mathrm{m}$ above the exit surface (slice B). In slice C, there are three stripes in the center and they appear to be interference fringes caused by crossover beams. After that, the crossover beams separate into further distances as seen in slice D. However, at 520 and $467 \mathrm{~nm}$ illumination, the transmission light separates from the beginning to the end, and there is no crossover observed during FDTD simulations [Figs. 4(c) and 4(e)] or experimentally. When we compare FDTD simulations [Figs. 4(a), 4(c), and 4(e)] with the experimental results (Fig. 2), we find good agreement at every cross section.

One interesting observation worth highlighting is that the two categories of beaming situations can be recognized in the pictures by distinguishing whether the emerging beams overlap or not [Figs. 4(a) and 4(c), respectively]. This interesting beaming feature can be explained by a surface plasmon diffraction theory. As stated in Eq. (2), when $k_{s p}$ is smaller than the grating vector $2 \pi / \Lambda$, the in-plane component of the wave vector of diffracted light $k_{0} \sin \theta$ is negative. In other words, the projected direction of the diffracted beam is opposite that of the SP propagation. Thus, accordingly we can define the beaming angle as being negative [Fig. 4(b)]. In such a situation, the two emerging beams overlap to form a scissorlike beaming pattern. Conversely, when the beaming angle is positive, the beams will propagate without intersecting [Fig. 4(d)]. Consequently, this feature indicates the inherent grating-diffraction nature of the beaming phenomenon. In addition, with such evidence, it also makes stronger the argument for our theory.

In conclusion, we propose that directional beaming is essentially an alternative form of surface plasmon diffraction. Moreover, since multiple scattering effects affect the surface 
plasmon dispersion relationship significantly, the beaming direction can be determined by the full interaction of the light beams and grating system, with primary emphasis on the grating topography such as period, shape, groove depth, etc. ${ }^{18-22}$ We know that the efficiency of the beaming and its degree of angular divergence are influenced by these factors as well. In addition, our proposed theoretical scheme can be both verified experimentally and numerically. Thus, we have excellent proof that our surface plasmon diffraction theory is closely related to the dynamics governing directional beaming.

Once we understand the phenomenon of beaming, we can control the beaming of light beams in the microscopic world. Along with this configuration, various nano-optical research fields and technologies can be facilitated and amplified, such as the field of optical switches, optical sensors, optical lithography, optical storage, and optical microscopy, etc. An important recent development proves that surface mode diffraction occurs in photonic crystals. ${ }^{23,24}$ If we modulate the period of photonic crystal interface cylinders, we should expect to see the same diffraction results as we have suggested. With applications to such a broad range of diverse potential products, the importance of this line of research work can be clearly seen and felt.

We are grateful for the many valuable technical discussions with Dr. J.P. Chen of the Materials Research Laboratory (MRL) at Industrial Technology Research Institute (ITRI). This research was partially supported by ITRI's MRL program "Nanowriter and Subwavelength Surface Structure Design for Optical Applications," and by National Science Council Program "Developing Piezotransformers Optimized for Wave Energy Reciprocal (NSC 93-2622-E-002-003)," and by the Department of Industrial Technology (DOIT), Ministry of Economic Affairs under the Technology Development Program for Academia "Wireless Health Advanced Monitoring Bio-Diagnosis System.”
*Electronic address: cklee@mems.iam.ntu.edu.tw

${ }^{1}$ T. W. Ebbesen, H. J. Lezec, H. F. Ghaemi, T. Thio, and P. A. Wolff, Nature (London) 391, 667 (1998).

${ }^{2}$ H. F. Ghaemi, Tineke Thio, D. E. Grupp, T. W. Ebbesen, and H. J. Lezec, Phys. Rev. B 58, 6779 (1998); T. Thio, H. F. Ghaemi, H. J. Lezec, P. A. Wolff, and T. W. Ebbesen, J. Opt. Soc. Am. B 16, 1743 (1999); T. J. Kim, T. Thio, T. W. Ebbesen, D. E. Grupp, and H. J. Lezec, Opt. Lett. 24, 256 (1999); D. E. Grupp, H. J. Lezec, T. W. Ebbesen, K. M. Pellerin, and T. Thio, Appl. Phys. Lett. 77, 1569 (2000); A. Krishnan, T. Thio, T. J. Kim, H. J. Lezec, T. W. Ebbesen, P. A. Wolff, J. Pendry, and L. MartinMoreno, Opt. Commun. 200, 1 (2001).

${ }^{3}$ H. J. Lezec, A. Degiron, E. Devaux, R. A. Linke, L. MartinMoreno, F. J. Garcia-Vidal, and T. W. Ebbesen, Science 297, 820 (2002).

${ }^{4}$ A. P. Hibbins, J. R. Sambles, and C. R. Lawrence, Appl. Phys. Lett. 81, 4661 (2002).

${ }^{5}$ F. J. Garcia-Vidal, H. J. Lezec, T. W. Ebbesen, and L. MartinMoreno, Phys. Rev. Lett. 90, 213901 (2003).

${ }^{6}$ L. Martin-Moreno, F. J. Garcia-Vidal, H. J. Lezec, A. Degiron, and T. W. Ebbesen, Phys. Rev. Lett. 90, 167401 (2003).

${ }^{7}$ F. J. Garcia-Vidal, L. Martin-Moreno, H. J. Lezec, and T. W. Ebbesen, Appl. Phys. Lett. 83, 4500 (2003).

${ }^{8}$ K. S. Kunz and R. J. Luebbers, The Finite Difference Time Domain Method for Electromagnetics (CRC Press, Boca Raton, FL, 1993).

${ }^{9}$ A. Yariv and P. Yeh, Optical Waves in Crystals (Wiley, New York, 1984).

${ }^{10}$ H. Raether, Surface Plasmons on Smooth and Rough Surfaces and on Gratings (Springer-Verlag, Berlin, 1988).

${ }^{11}$ R. W. Wood, Philos. Mag. 4, 396 (1902); Phys. Rev. 48, 928
(1935).

${ }^{12}$ U. Fano, J. Opt. Soc. Am. 31, 213 (1941); A. Hessel and A. A. Oliner, Appl. Opt. 4, 1275 (1965).

${ }^{13}$ W. Rothballer, Opt. Commun. 20, 429 (1977).

${ }^{14}$ M. C. Hutley, Diffraction Grating (Academic Press, New York, 1982).

${ }^{15}$ T. K. Gaylord and M. G. Moharam, Proc. IEEE 73, 894 (1985).

${ }^{16}$ D. Z. Lin, L. B. Yu, C. K. Lee, C. S. Yeh, and C. L. Lin, Scanning 26, 73 (2004).

${ }^{17}$ E. D. Palik, Handbook of Optical Constants of Solids (Academic Press, Orlando, FL, 1985).

${ }^{18}$ A. Wirgin, Opt. Acta 27, 1671 (1980); 28, 1377 (1981).

${ }^{19}$ A. Marvin, F. Toigo, and V. Celli, Phys. Rev. B 11, 2777 (1975); R. I. Masel, R. P. Merrill, and W. H. Miller, ibid. 12, 5545 (1975); F. Toigo, A. Marvin, V. Celli, and N. R. Hill, ibid. 15, 5618 (1977); Bernardo Laks, D. L. Mills, and A. A. Maradudin, ibid. 23, 4965 (1981); M. Weber and D. L. Mills, ibid. 27, 2698 (1983); N. Garcia, Opt. Commun. 45, 307 (1983); N. E. Glass, M. Weber, and D. L. Mills, Phys. Rev. B 29, 6548 (1984).

${ }^{20}$ J. Chandezon, M. Dupuis, G. Cornet, and D. Maystre, J. Opt. Soc. Am. 72, 839 (1982).

${ }^{21}$ E. Popov and L. Mashev, Opt. Acta 33, 593 (1986); J. P. Plumey, B. Guizal, and J. Chandezon, J. Opt. Soc. Am. A 14, 610 (1997); G. Granet, ibid. 15, 1121 (1998).

${ }^{22}$ N. Garcia, V. Celli, N. R. Hill, and N. Cabrera, Phys. Rev. B 18, 5184 (1978).

${ }^{23}$ P. Kramper, M. Agio, C. M. Soukoulis, A. Birner, F. Muller, R. B. Wehrspohn, U. Gosele, and V. Sandoghdar, Phys. Rev. Lett. 92, 113903 (2004).

${ }^{24}$ E. Moreno, F. J. Garcia-Vidal, and L. Martin-Moreno, Phys. Rev. B 69, 121402(R) (2004). 